\title{
Cómo integrar los componentes biofísicos con los socioculturales y económicos en la caracterización y análisis espacial de los sistemas agrarios.Propuesta metodológica Caso: municipio de Cota, Cundinamarca
}

\author{
Sandra Lucía Ruiz Rodríguez*
}

Resumen. El presente artículo expone de manera sucinta una propuesta metodológica para caracterizar y analizar espacialmente los sistemas agrarios. Esta metodología ha sido aplicada para el caso de los sistemas agrarios del municipio de Cota. La importancia de esta metodología radica en que logra a través del enfoque sistémico integrar operativamente los componentes y factores físicos, bióticos y socioculturales para conocer y entender la estructura, función y manejo de los sistemas agrarios. Así mismo, permite clasificar y representar espacialmente unidades sociales homogéneas y unidades socioeconómicas, que posteriormente se integran a las condiciones biofísicas para lograr representar espacial e integralmente los diferentes sistemas que se presentan en un espacio agrario, en particular en el municipio de Cota. Palabras clave: caracterización, enfoque sistémico, componentes, factores y variables.

* Ingeniera Agrícola, Universidad Nacional de Colombia. Magíster en Geografía con énfasis Ordenamiento Territorial, EPG, convenio UPTC - IGAC. Especialista en Docencia Universitaria. 


\begin{abstract}
This article exposes of a summary way a methodological proposal to characterize and analize spatialy the agrarian systems. This methodology has been applied to the case of agrarian systems of the Cota municipium in Cundinamarca. The detail of the methodology and the results of the application of it, are registered in the entitled investigation "characterization and spatial analysis of the agrarian systems in the Cota municipium in Cundinamarca".

The importance of this methodology is that it achieves, through of systemic approach, integrates operatively, the components and physical, biotic and sociocultural factors to know and two understand the structure, function and management of the agrarian systems. Also, it allows to classify and represent spatialy, homogeneous social units and socioeconomic units, that after they integrate into the biophysics conditions to achieve representing spacial and integrally the different systems that appear in an agrarian space, especifically, in the Cota municipium in Cundinamarca. Key words: characterization, systemic approach, components, factors and variables.
\end{abstract}

Resumé. Cet article expose de manière suscinte une proposition méthodologique pour caractériser et analyser territorialement les systèmes agraires. Cette méthodologie a été appliquée dans le cas des systèmes agraires de la municipalité de Cota (Cundinamarca). Le détail de cette méthodologie ainsi que les résultats de l'application de celle ci, se trouvent enregistrés dans l'investigation intitulée "Caractérisation et analyse territoriale des systèmes agraires présents dans la municipalité de Cota - Cundinamarca". L'importance de cette méthodologie réside dans le fait, qu'au travers de l'optique systématique, elle arrive a intégrer opérativement les composants et facteurs physiques, biotiques et socioculturels pour connaître et comprendre la structure, la fonction et l'utilisation des systemes agraires. Ainsi elle permet de classifier et représenter dans l'espace des unités sociales homogènes, et des unités socioéconomiques qui ultérieurement s'intègrent aux condition biophysiques por arrivées á représenter les différents systèmes qui se trouvent dans un espece agraire, en particulier dans la municipalité de Cota (Cundinamarca). Mots Clées: Caractérisation, optique systématique, composants, facteurs et variables. 


\section{Introducción}

Entender cómo es la estructura y función de los sistemas, entre éstos los agrarios, resulta a menudo complicado en razón a la diversidad de factores y variables que juegan en el proceso productivo.

Son diversos los enfoques y métodos que las ciencias físicas y biológicas han utilizado para analizar los fenómenos de manera integral. Con el invento del microscopio, ya hace más de un siglo, la investigación en las ciencias físicas y biológicas hizo énfasis en un enfoque atomicista, en el cual se analizaba un fenómeno por un método que se basaba en la descripción y análisis de los elementos que conformaban el fenómeno. Así, los biólogos empezaron a describir la anatomía de las plantas y los animales, los físicos a describir moléculas y electrones; todo ello, con el objeto de entender cómo funcionaban estos fenómenos basándose en un análisis de los componentes que los conforman (Hart, 1980).

El enfoque de sistemas surgió de comprender que con la sola descripción de los componentes de un fenómeno, no es suficiente para explicarlo, también se necesita saber la relación entre los componentes, en otras palabras la función de un sistema, depende de su estructura. Por tanto, analizar cualquier fenómeno como un sistema es entender la relación entre su estructura y su función.
Pese a la importancia y aceptación del enfoque de sistemas, los estudios y proyectos desarrollados bajo esta orientación se han quedado cortos en el análisis integral de los fenómenos humanos y ambientales, lo anterior no porque el enfoque como tal no lo permita, sino porque se han reducido los postulados del concepto de sistema (Berdegué y Escobar, 1995). Algunos estudios y proyectos centran sus objetivos en incrementar la producción agropecuaria, en tanto que otros, ignoran diversas relaciones macromicro entre los sistemas agrarios y se restringe el campo de operaciones a la finca. Por otro lado, es evidente la dificultad de analizar y de manejar operacionalmente las interrelaciones entre los factores económicos, sociales, culturales y políticos, lo que ha originado un énfasis en el análisis de factores físicos y algunos económicos (Berdegué y Escobar, 1995). Así mismo, evaluaciones adelantadas por varios organismos nacionales e internacionales (CORPOICA, GTZ; RIMISP, entre otras.), concluyen que buena parte de los proyectos agropecuarios que se implementan, no consideran las verdaderas necesidades y problemas de la población, pues los estudios previos a la implementación, hacen especial énfasis en las variables ecológicas, olvidando que los productores, cuando seleccionan una $\mathrm{u}$ otra actividad en su finca, tienen presente también condiciones económicas, sociales y culturales. Esto ha llevado a incontables fracasos en la implementación de proyectos. 
Se suma a lo anterior, el hecho de que la información disponible para abordar el análisis de los sistemas agrarios, se presenta en la mayoría de los casos de forma sectorizada y pocas veces se encuentra georeferenciada, lo cual dificulta aún más un análisis integral.

Lo arriba expresado, fue el estímulo para el desarrollo de la presente metodología, aplicada al caso del municipio de Cota - Cundinamarca, la cual busca integrar operativamente las relaciones y variables económicas, sociales, culturales y biofísicas para facilitar el conocimiento y organización de los sistemas agrarios que se presentan en un espacio determinado. Pretende además identificar los factores y variables que determinan en mayor grado la diversidad de sistemas agrarios; así como los espacios con características relativamente homogéneas y zonificar y espacializar dichos sistemas agrarios.

\section{Metodología}

\section{Enfoque de sistemas}

Partiendo del concepto de sistema como orden dinámico de partes en interacción mutua, un sistema agrario es un conjunto de elementos naturales y humanos en interacción dinámica en función de un objetivo: obtener productos $\mathrm{y}$ beneficios (Domínguez, 1992).

Todo sistema tiene una estructura y una función. La primera está vinculada con el arreglo o forma como se organizan sus componentes, en tanto que segunda está estrechamente relacionada con la estructura e indica el proceso interno para transformar las entradas en salidas (Rodríguez, 1998). Así mismo, está conformado por una serie de elementos: los límites, las entradas y salidas, los componentes y la interacción entre componentes.

Los límites, son entendidos como la línea divisoria entre dos espacios, cada uno con características internas homogéneas y lo suficientemente heterogéneos respecto a cualquier otro, de tal forma que sean fácilmente diferenciables (Rodríguez, 1998). Lo anterior es un principio fundamental para establecer y espacializar a nivel del municipio de Cota, los sistemas agrícolas, pecuarios y forestales, los cuales se localizan en diferentes espacios físicos y con particulares condiciones sociales, culturales y económicas.

Las entradas y salidas son los flujos que entran y salen de la unidad. Así, las entradas o insumos de un sistema agrario, son constituidos por flujos de recursos, productos, energía, conocimientos e información provenientes del ambiente externo, mientras que las salidas se consideran como el flujo de productos, materia prima, insumos, información y materiales o recursos que salen del sistema.

Los componentes son las unidades productivas, humanas, físicas y de infraestructura del sistema. Hacen

10 Sandra Lucía Ruiz Rodríguez 
referencia al conjunto de las diversas maneras como éstos se relacionan en el tiempo y en el espacio, así como a la naturaleza de estas relaciones; es lo que proporciona las características de estructura a la unidad (Figura 1). Los componentes clasificados en los sistemas agrarios del área estudiada fueron el componente físico, biótico, sociocultural y económico.
Fundamentados en las bases teóricas, desarrollos conceptuales y aplicación de esquemas de trabajo apropiados para integrar los componentes físicos, bióticos, socioculturales y económicos como un todo (los principios básicos de teoría de sistemas), se procedió a la identificación, clasificación y análisis de los diferentes sistemas agrarios del municipio de Cota.

Figura 1. Elementos de los Sistemas Agrarios

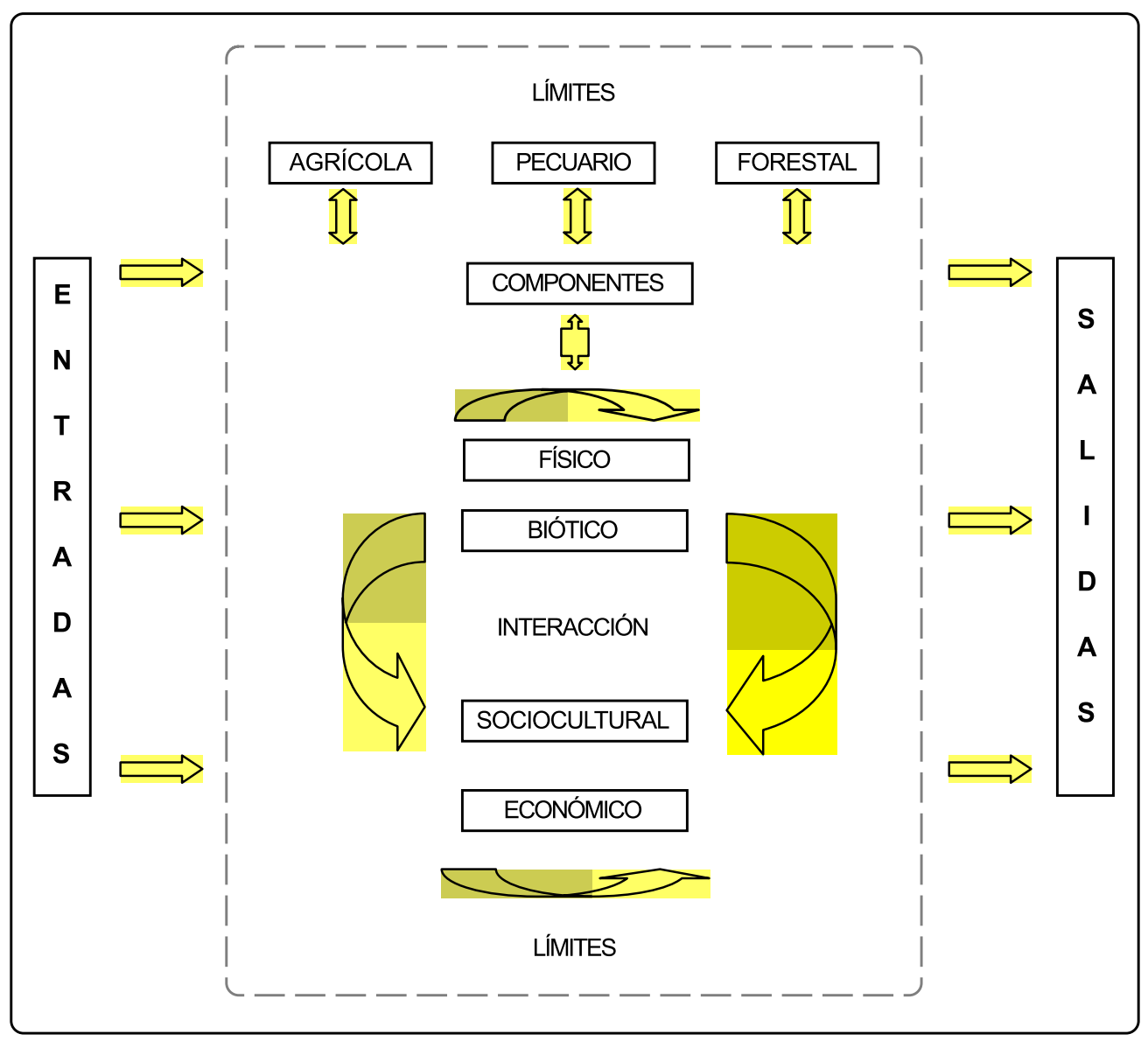

Fuente: Diseño de la autora, con base en las definiciones antes anotadas. 
El proceso metodológico que se describe a continuación, contempla una serie de pasos que conducen de forma clara $y$ ordenada a la caracterización y espacialización de dichos sistemas:

a) Definición de componentes, factores y variables. La identificación y clasificación de los sistemas agrarios, se desarrolló con base en la definición de los componentes, factores y variables tanto biofísicos, como socioculturales y económicos:

Componente físico. Se definió a través de los factores clima, geología, geomorfología y suelos, los cuales se analizaron uno a uno, para posteriormente integrarse y definir espacios homogéneos físicos.

Componente biótico. Constituido por los factores flora y fauna. La identificación y clasificación de este componente se fundamentó en la especialización y objetivo productivo de la cobertura vegetal. La observación de este aspecto condujo a concluir que en el municipio de Cota se encuentran sistemas agrícolas, sistemas pecuarios y sistemas forestales.

Componente sociocultural y económico. El estudio y la zonificación socioeconómica y cultural partió de cuatro factores estructurales: el demográfico, el social, la infraestructura física y la estructura productiva. De cada factor se definieron variables que a juicio de la autora son determinantes para la caracterización y análisis de los sistemas agrarios. Así mismo, se estableció qué era importante estudiar de cada variable y para qué.

El factor demográfico se definió con las siguientes variables:

Dinámica demográfica: permite establecer el origen y procedencia de la población y el crecimiento poblacional. Lo anterior permite proyectar porcentajes de población con vocación agraria.

Densidad poblacional: establece grupos etáreos y población econó-micamente activa por sexo orientado a identificar la oferta laboral en relación con las actividades agrarias adelantadas por la población.

Distribución espacial de la población: identifica zonas con mayor o menor concentración poblacional, principalmente con fines de disponibilidad de mano de obra.

Las variables estudiadas dentro del factor social fueron:

Condiciones culturales: determina la existencia o no de una vocación agropecuaria, hacia el trabajo comunitario, una valoración de los recursos naturales y un arraigo hacia su municipio y región.

Condiciones educativas: evalúa, por un lado, el nivel de escolaridad como alternativa de la población para continuar asentada en una zona $y$, por otro 
lado, si el tipo de capacitación dada está relacionada con el desarrollo agrario del municipio.

Condiciones de salud: establece la relación entre el estado de salud de la población con las prácticas productivas e identifica si la actividad agraria realizada permite a los habitantes agrarios suplir sus necesidades básicas.

La infraestructura física: evalúa tanto la cantidad como la calidad de la infraestructura apta para la producción y el mercadeo. Dentro de este factor se estudiaron las siguientes variables:

- Vías (estado y clase).

- Transporte y comunicación.

- Cercanía a los centros de consumo.

- Funcionamiento espacial.

- Costos del transporte.

La estructura productiva está determinado por las siguientes variables:

Tamaño de la tierra: establece la relación entre el tamaño de los predios dedicados a la actividad agraria y la eficiencia productiva, económica y ambiental (manejo y conservación de los recursos naturales: desgaste de la tierra y agua).

Tenencia de la tierra: identifica los vínculos de propiedad a partir de tres categorías: propietarios, arrendatarios y aparceros. Además, permite evaluar el arraigo hacia la explotación agraria, los recursos naturales o si el interés es solamente de aumentar la producción y la ganancia.

Actividad agraria: se definieron tres tipos de actividades agrarias predominantes a partir del uso y el aprovechamiento que la población hace de la tierra y de la cobertura vegetal: agrícolas, pecuarias y forestales; estas se analizaron a partir de los siguientes aspectos: objetivo de la producción, uso $\mathrm{y}$ origen de la fuerza de trabajo, manejo tecnológico, origen de los insumos, riesgo de incertidumbre, destino de la producción, administración del sistema, procesamiento, crédito, fijación de precios, salarios, ingresos netos, apoyo científico y asistencia técnica, incentivos financieros, subsidios agrícolas y efectos ambientales.

b) Captura, tratamiento y evaluación de la información. La información recogida proviene de fuentes primarias y secundarias. Por el mismos carácter de algunas variables estructurales (demográfico, social, infraestructura física y estructura productiva) se optó por realizar una labor de campo, para lo cual se seleccionó una muestra representativa de la población, de la cual mediante entrevistas estructuradas y no estructuradas, las técnicas del D.R.P. y charlas informales, se obtuvo la información necesaria.

Recogida la información primaria y secundaria, el tratamiento seguido consistió en el ordenamiento, 
clasificación, codificación de los datos, homogeneización de formatos, escalas y fuentes, la aplicación y análisis de procedimientos estadísticos, así como la transformación de variables a categorías, medios que facilitan el proceso de descripción, análisis integral y la síntesis final.

Tratamiento de la información de los componentes físico y biótico. El tratamiento de este componente consistió en analizar los factores y variables bióticos, con el fin de hallar aquellos que dadas sus características internas, guardan cierta capacidad discriminatoria y logran agrupar unidades homogéneas. Esto permitió establecer que la fisiografía tiene capacidad discriminatoria y permite clasificar grupos con cierta homogeneidad, ya que según las unidades de paisaje se pueden observar diferencias significativas en la conformación de los sistemas agrarios, en el objetivo de los productores, en las prácticas de manejo y en el establecimiento de relaciones con el entorno. Así, dicho factor ${ }^{1}$ llevó a la definición de sistemas de producción localizados en diferentes paisajes y geoformas.

El componente biótico y el físico se reclasificaron $y$ se cruzaron cartográficamente para generar las unidades biofísicas homogéneas que se representan en el mapa de unidades biofísicas homogéneas.
Tratamiento de los componentes sociocultural y económico. Una vez ordenada, clasificada y codificada la información, se procedió al análisis y descripción de cada variable y de cada factor demográfico, social y económico. El procedimiento se realizó con la división de los factores en dos grupos principales: de un lado, el sociocultural conformado por demografía, educación, salud y condiciones culturales y, de otro, el económico (estructura productiva e infraestructura física). La descripción y análisis evidenciaron unidades con ciertas características de homogeneidad que transformadas en categorías facilitaron una clasificación por variables $y$ por factores generando una zonificación social o de calidad de vida y una económica; éstas posteriormente se integraron para dar como resultado la zonificación socioeconómica del municipio.

Detalle del tratamiento y evaluación de los factores sociales para la zonificación. Con el fin de generar la zonificación social, al componente sociocultural se le adicionó el valor de la tierra obtenido de las zonas geoeconómicas elaboradas por el Instituto geográfico Agustín Codazzi IGAC (actualizado para Cota en el año 2000), para efectuar el avalúo de los predios y que se representan cartográficamente.

\footnotetext{
1 El Plan Básico de Ordenamiento Territorial de Cota, 2000, se basó en el análisis fisiográfico para adelantar la zonificación ambiental, esta zonificación permitió determinar las unidades de paisaje que se describen en esta investigación.
}

14 Sandra Lucía Ruiz Rodríguez 
Se optó por el valor de la tierra ya que es un factor con capacidad discriminatoria e indicador indirecto de calidad de vida, entendiendo que si un predio y una zona están avaluados por ejemplo en $\$ 40.000 .000$ (para el año 2000) la hectárea, quiere decir que el propietario tiene los medios económicos suficientes y una calidad de vida que le permite pagar y responder por ese valor; caso contrario, si un predio está avaluado, por ejemplo, en $\$ 200.000$ la hectárea, se puede argumentar que el propietario y su familia tienen una baja capacidad económica que compromete la calidad de vida.

Cabe resaltar que las zonas homogéneas geoeconómicas son el resultado de la integración de variables biofísicas, de infraestructura y de calidad de la vivienda, las cuales son tenidas en cuenta por Planeación Nacional para calcular el índice de calidad de vida (educación, capital humano, calidad de la vivienda, servicios, tamaño y composición familiar). Es decir, en esta investigación el valor de la tierra se tomó como un primer indicador de calidad de vida, el cual se enriqueció correlacionando e integrando las variables densidad y distribución de la población, vocación agrope-cuaria, vocación hacia el trabajo comunitario, valoración hacia los recursos naturales, arraigo, educación y salud. Las anteriores variables se analizaron una a una y se clasificaron y correlacionaron a partir de la definición de rangos que indican el grado de afectación de cada variable sobre las condiciones de vida de los productores agrarios (tabla 1$)^{2}$. Esto facilitó la espacialización de cada una de ellas y el posterior cruce cartográfico que generó una serie de unidades clasificadas en calidad de vida alta, media y baja; partiendo de dos variables limitantes o con capacidad de discriminación, la salud y la densidad poblacional, considerando rangos dadas a ellas y la implicación que tienen en la calidad de vida de la población. En el cuadro 1 se muestran algunas de las combinaciones que se presentaron al integrar las variables. El proceso anterior arrojó los resultados que se representaron en el mapa social o de calidad de vida.

Tratamiento del componente económico. Dentro del componente económico como ya se señaló, se estudió la infraestructura física, integrada por el sistema vial, el transporte y el funcionamiento espacial del municipio y la estructura productiva, que contempla el uso, tamaño y tenencia de la tierra y la actividad agraria como tal.

Los procesos de análisis integrado para la zonificación, requieren de la selección de factores y variables con capacidad de clasificar grupalmente unidades espaciales homogéneas, que compartan ciertas condiciones que las diferencian de las demás.

2 La definición de los rangos de las demás variables, puede consultarse en el documento de investigación referenciado inicialmente. 
Dentro de las variables económicas estudiadas, se encontró que el tamaño predial y la tenencia de la tierra, en el municipio de Cota, tienen la capacidad de clasificar grupos homogéneos y diferenciar la intensidad en el uso del suelo y además, dan indicios del grado de productividad alcanzado.
Las actividades agrarias típicas del área de estudio (agrícola, pecuaria y forestal), así como el tamaño predial, fueron las variables y factores diferenciadores para la configuración de los espacios homogéneos. Así se clasificaron espacios dedicados a la práctica agrícola, pecuaria y forestal, orientados a la producción de

Tabla 1

Definición de rangos clasificatorios de las variables sociales y culturales

Zona Geoeconómica: Valor de la tierra

\begin{tabular}{|l|l|}
\hline Grado & DEFINICIÓN \\
\hline Alto & Zona donde el valor de la tierra oscila entre $\$ 27$ y $\$ 40$ millones la hectárea. \\
\hline Medio & Zona donde el valor de la tierra oscila entre $\$ 12$ y $\$ 18$ millones la hectárea. \\
\hline Bajo & Zona donde el valor de la tierra oscila entre $\$ 130$ y $\$ 500$ mil pesos la hectárea. \\
\hline
\end{tabular}

Densidad y distribución de la población

\begin{tabular}{|l|l|}
\hline Grado & DEFINICIÓN \\
\hline Alto & $\begin{array}{l}\text { Zona con mayor número de habitantes, mayor cantidad de viviendas por hectárea, } \\
\text { y mayor cantidad de predios por hectárea. }\end{array}$ \\
\hline Medio & $\begin{array}{l}\text { Zona donde existe una proporción media de habitantes, de viviendas por hectárea } \\
\text { y de predios. }\end{array}$ \\
\hline Bajo & $\begin{array}{l}\text { Zona con menor proporción de habitantes, de vivienda por hectárea y menor } \\
\text { cantidad de predios por hectárea. }\end{array}$ \\
\hline
\end{tabular}

Vocación Agropecuaria

\begin{tabular}{|l|l|}
\hline Grado & \multicolumn{1}{c|}{ DEFINICIÓN } \\
\hline Alto & $\begin{array}{l}\text { Zona donde se localiza la población más autóctona y la principal actividad es la } \\
\text { horticultura tradicional }\end{array}$ \\
\hline Medio & $\begin{array}{l}\text { Zona en donde se ha localizado la población que migró más recientemente y su } \\
\text { principal actividad es la agrícola o pecuaria. }\end{array}$ \\
\hline Bajo & $\begin{array}{l}\text { Zona en donde se ha localizado la población que migró más recientemente y que } \\
\text { están dedicadas a actividades diferentes a la agrícola o pecuaria (industria, } \\
\text { recreación, vivienda campestre, servicios, protección - recuperación). }\end{array}$ \\
\hline Salud & \multicolumn{1}{|c|}{ DEFINICIÓN } \\
\hline Grado & $\begin{array}{l}\text { La actividad agropecuaria no le afecta la salud de manera significativa y le suple sus } \\
\text { necesidades básicas y las de su familia. }\end{array}$ \\
\hline Medio & $\begin{array}{l}\text { La actividad agropecuaria le afecta la salud, pero le suple sus necesidades básicas y las de } \\
\text { su familia. }\end{array}$ \\
\hline Bajo & $\begin{array}{l}\text { La actividad agropecuaria le afecta la salud y no le suple sus necesidades básicas y las de su } \\
\text { familia. }\end{array}$ \\
\hline
\end{tabular}

Fuente: Rangos definidos por la autora con base en la definición dada a cada una de las variables estudiadas. 


\section{Cuadro 1}

Relaciones entre las variables sociales y determinación de la calidad de vida

\begin{tabular}{|c|c|c|c|c|c|c|}
\hline $\begin{array}{l}\text { Calidad } \\
\text { de vida }\end{array}$ & $\begin{array}{l}\text { Precio de } \\
\text { de tierra }\end{array}$ & $\begin{array}{l}\text { Densidad } \\
\text { de la } \\
\text { Población }\end{array}$ & $\begin{array}{l}\text { Vocación } \\
\text { Agrope- } \\
\text { cuaria }\end{array}$ & $\begin{array}{c}\text { Vocación } \\
\text { Trabajo } \\
\text { Comunitario }\end{array}$ & $\begin{array}{l}\text { Valoración } \\
\text { Recursos } \\
\text { Naturales }\end{array}$ & Salud \\
\hline \multirow{5}{*}{ ALTA } & \multirow[b]{2}{*}{ ALTO } & Media & Media & Media & Media & Alta \\
\hline & & Baja & Baja & Baja & Alta & Alta \\
\hline & \multirow{4}{*}{ MEDIO } & Media & Baja & Baja & Alta & Alta \\
\hline & & Baja & Baja & Baja & Alta & Alta \\
\hline & & Baja & Media & Media & Media & Alta \\
\hline BAJO & & Baja & Media & Media & Media & Alta \\
\hline \multirow{12}{*}{ MEDIA } & \multirow{12}{*}{ ALTO } & Alta & Baja & Baja & Media & Media \\
\hline & & Alta & Media & Media & Media & Alta \\
\hline & & Media & Media & Media & Baja & Media \\
\hline & & Media & Alta & Alta & Baja & Media \\
\hline & & Media & Baja & Baja & Media & Media \\
\hline & & Alta & Alta & Alta & Baja & Media \\
\hline & & Alta & Alta & Alta & Media & Alta \\
\hline & & Alto & Bajo & Bajo & Alta & Alta \\
\hline & & Alto & Bajo & Bajo & Bajo & Alta \\
\hline & & Bajo & Bajo & Bajo & Medio & Medio \\
\hline & & Bajo & Medio & Medio & Bajo & Medio \\
\hline & & Bajo & Alto & Alto & Bajo & Medio \\
\hline
\end{tabular}

hortalizas, flores, ganadería bovina / lechería, con destino a la producción comercial y comercial empresarial, y los forestales orientados a la producción de madera y demás subproductos $\mathrm{y}$, por último, de producción-protección de servicios ambientales y reglamentados según $\mathrm{PBOT}, 2000$ como áreas de protección de acuíferos, suelos y biodiversidad.

Igualmente, se relacionaron con el uso intensivo de mano de obra e insumos, así como del suelo, localizados en áreas de micro-minifundio, microfundio, minifundio, pequeña, mediana y gran propiedad, con similares condiciones de infraestructura, tanto vial como de servicios.

Con base en lo anterior se identificaron diversos espacios homogéneos económicos los cuales se representan en el mapa de unidades económicas.

La zonificación económica se integró, como ya se indicó, a la zonificación 
social o de calidad de vida, lo que originó unidades o zonas con un alto grado de homogeneidad en cuanto a sus componentes sociales (demográficos, culturales, educativos y de salud) y económicos (infraestructura física y estructura productiva), siendo la estructura productiva / actividad agraria la variable de mayor relevancia para la espacialización de cada una de las zonas. Esta zonificación es presentada en el mapa de unidades socioeconómicas homogéneas. Las zonas homogéneas socioeconómicas, una vez se incorpora en su análisis y descripción las condiciones biofísicas, adquieren una mayor claridad de expresión, reconocimiento descriptivo y localización espacial. Es decir, se obtiene la caracterización y zonificación de dichos sistemas, ya que se ha logrado un conocimiento integral de las circunstancias físicas, bióticas, económicas y socioculturales, necesarias para entender y formular hipótesis acerca de la estructura, función y manejo y la razón de ser de los sistemas agrarios (Rodríguez, 1993).

La aplicación de la anterior metodología al caso de Cota permite presentar el mapa de sistemas agrarios. Para efectos de este artículo, se describe uno de los subsistemas encontrados, en la que puede apreciarse la caracte- rización desde las diferentes dimensiones física, biótica, económica y social y su integración, y las ventajas que conlleva el considerar en conjunto éstas dimensiones para tener una concepción más explícita del funcionamiento y estructura del subsistema: ${ }^{3}$

Horticultura intensiva en abanicos y calidad de vida baja. Localizada en las estribaciones de la montaña en áreas que pertenecen a las veredas La Moya, Cetime y parte de Abra. Los suelos son moderadamente profundos y profundos, bien drenados, con texturas medias y gruesas. La fertilidad es baja a media.

Son zonas susceptibles a la erosión laminar principalmente. La actividad hortícola se desarrolla de manera intensiva, tanto en mano de obra como en el uso de insumos. Prevalece el micro minifundio $\left(1-2.000 \mathrm{~m}^{2}\right.$. $)$ y el microfundio ( 2.001 a $5.000 \mathrm{~m}^{2}$.) lo que indica que hay sobreexplotación del suelo y por ende, deterioro progresivo del mismo.

Las hortalizas se siembran entre dos y tres veces al año, ya que en los periodos secos no se pueden regar los cultivos, pues el agua subterránea y superficial es escasa (las características hidrogeológicas indican una importancia moderada y las fuentes superficiales se ha secado).

3 El detalle de la caracterización y aplicación de los sistemas agrarios del municipio de Cota puede consultarse en el docmento ya referenciado.

I 8 Sandra Lucía Ruiz Rodríguez 
El Plan Básico de Ordenamiento Territorial (PBOT) de Cota -2000reglamenta el uso del suelo de esta unidad como de manejo integrado, con el uso principal en conservación y protección, compatible con uso recreacional pasivo. Se prohíben mayores subdivisiones de las unidades agrarias. El uso agrícola, la captación de agua y la implementación de mayor infraestructura está condicionados.

En esta unidad se encuentra buen porcentaje de la población indígena perteneciente al resguardo cuya vocación es agropecuaria con predominio del trabajo comunitario. La base económica la constituye la agricultura-horticultura asociada con algunos relictos de vegetación arbustiva y arbórea dispersa. Esta actividad no provee los recursos económicos nece-sarios para que el agricultor y su familia suplan sus necesidades básicas y por tanto, el productor se ve obligado a buscar otras alternativas entre las que se encuentran el jornaleo y las "socias" para cultivar en el mismo municipio o de otros.

Es una comunidad muy arraigada tanto a sus costumbres como a su municipio y región. El nivel de escolaridad promedio es la primaria y se interesan por una capacitación orientada hacia el manejo integral de la finca y la agricultura orgánica. En cuanto a las condiciones de salud, se puede afirmar que dentro del trabajo agrícola el manejo indistinto de agroquímicos está afectando negativamente la salud humana y los recursos naturales.
Dadas las anteriores características se puede concluir que esta unidad no permite un desarrollo agrícola hortícola como el que se está dando, no sólo por las condiciones físicas que la caracterizan y por el manejo dado, sino por las disposiciones reglamentadas por el P.B.O.T. es conveniente identificar alternativas económicas para potenciar la vocación agropecuaria y organizativa de sus pobladores, que sirvan además para suplir sus necesidades básicas.

\section{Conclusiones}

El enfoque de sistemas y la metodología desarrollada permitieron la integración operativa de los componentes físicos, bióticos y socioculturales, lo que se convierte en un paso más hacia la integración operativa especialmente de la dimensión social a lo biofísico y económico. Se espera que esta metodología sea mejorada $y$ enriquecida para avanzar en ese cuello de botella, por tanto queda abierta la discusión frente a esta propuesta, así como también, está al servicio de otros municipios o instituciones que a bien consideren implementarla y mejorarla.

Los factores y variables definidos y estudiados permitieron explicar la estructura, función y el manejo de los sistemas agrarios presentes en el municipio de Cota. Dentro de cada factor $\mathrm{y}$ dadas las condiciones $\mathrm{y}$ configuraciones de los sistemas agrarios 
se encontraron variables con mayor capacidad de discriminación o mayor grado de diversidad que tipifican los distintos sistemas. En el factor físico la fisiografía fue determinante para entender la localización y distribución de los sistemas, mientras que en lo social, las condiciones de salud y la densidad poblacional, facilitaron diferenciar unidades sociales con características particulares que obedecen al comportamiento de los diferentes grupos sociales asentados allí; en lo económico, la actividad agraria, el manejo tecnológico y el tamaño predial, fueron las variables que permitieron definir espacios económicos con características de homogeneidad.

La zonificación y representación espacial de los diferentes aspectos físico- bióticos, económicos y sobre todo los sociales, facilitan el análisis, explicación y posterior integración, para lograr interpretación de las relaciones espaciales y funcionales que configuran unos espacios homogéneos particulares en este caso agrarios.

El estudio permitió establecer diferencias importantes entre los sistemas productivos encontrados a partir de su estructura, función, relaciones y calidad de vida, lo cual se convierte en insumo para acciones orientadas ya sea hacia la planificación regional o local, a la organización de la comunidad, a la investigación y transferencia de tecnología, que apunten a un mayor impacto en términos de productividad $y$ sostenibilidad y por último, que guarden total relación no sólo con las condiciones físicas sino sociales y económicas de cada sistema y actividad agraria desarrollada en el municipio.

El municipio de Cota tiene características básicamente rurales, de tal manera que su base económica aún se soporta en las actividades agropecuarias; sin embargo, la presión que genera su cercanía a Bogotá, expresada en la creciente especulación y fragmentación de la tierra, la ocupación del suelo en servicios educativos, recreacionales (clubes privados, restagurantes, entre otros), industria, comercio y construcción, está amenazando con ocupar y acabar los suelos de alta capacidad agropecuaria que tiene el municipio. De igual manera, la población más autóctona estaría no sólo perdiendo su vocación agropecuaria sino que además se estaría aumentando el desempleo, pues la actividad agrícola hortícola es una de las que más demanda mano de obra. 


\section{Bibliografía}

ANDRADE, Ángela. Aspectos conceptuales y metodológicos para el diseño de las bases de datos utilizados en el análisis de sistemas agrarios. Revista SIG- PAFC, No. 10-11. Santafé de Bogotá, Colombia, 1996. 86 p.

Alcaldía Municipal de Cota. Plan básico de ordenamiento territorial - análisis territorial municipal, documento de soporte técnico. Cota, Cundinamarca, 2000. 99 p.

BERDEGUÉ, Julio y ESCOBAR, Germán. Nuevas direcciones del enfoque de sistemas para la modernización de la agricultura campesina en América Latina. RIMISP, Santiago de Chile, 1995. p. 13-43.

Corporación Colombiana de Investigación Agropecuaria, CORPOICA. Caracterización biofísica, tecnológica y socioeconómica de los sistemas de producción de las islas Providencia y Santa Catalina. Montería, Colombia, 1998. 45 p.

DEPARTAMENTO NACIONAL DE PLANEACIÓN - DIRECCIÓN DE DESARROLLO AGRARIO/ECONOMETRIA S.A. Evaluación ambiental de los proyectos piloto de las alianzas productivas para la paz. Hortalizas para el municipio de Cota. Vol. I. Bogotá D.C., 2000. 100 p.

DIAZ, Ramón. Geografía y agricultura - componentes de los espacios agrarios. Cuadernos de estudio No.4. España, 1990. 128 p.

ESCOBAR, Germán. Investigación con enfoque de sistemas en la agricultura y el desarrollo rural. RIMISP. Santiago de Chile, 1995. p. 99 -109.

FEDERACIÓN COLOMBIANA DE GANADEROS - FEDEGAN. La ganadería Bovina en Colombia 2000 - 2001. Bogotá D.C., 2002. 17 p.

FLOREZ, A. La Teoría General de los Sistemas. Cuadernos de Geografía. Vol IV No. 1 2. Universidad Nacional de Colombia, 1993. 138 p.

Perspectiva Geográfica 21 
GARCIA, D. y otros. Geografía rural. Espacios y sociedades No. 10. Editorial síntesis. Madrid - España, 1995. 235 p.

HART, Robert. Sistemas. CATIE, 1980.

INSTITUTO GEOGRÁFICO AGUSTÍN CODAZZI. Guía metodológica para la formulación del plan de ordenamiento territorial Municipal. Santafé de Bogotá, Colombia, 1997. $186 \mathrm{p}$.

Zonificación socioeconómica de la cuenca hidrográfica del río Sinú (parte media y baja) departamento de Córdoba. Santa Fe de Bogotá, Colombia, 1998. 140 p.

INSTITUTO COLOMBIANO AGROPECUARIO - ICA -. La ganadería de la leche en la meseta central de la sabana de Bogotá. Bogotá D.C., 1988. 151 p.

PÉREZ- ARBELÁEZ, Enrique. Plantas Útiles de Colombia. 14 ed. Medellín: Víctor Hugo, 1990. $832 \mathrm{p}$.

RODRÍGUEZ QUIJANO, Pedro. Sistemas de producción. Conceptos y métodos de aplicación. Santa Fe de Bogotá, Colombia, 1993. 102 p.

. Propuesta metodológica para la caracterización de sistemas productivos agropecuarios a nivel nacional, regional y local. Corporación Colombiana de Investigación agropecuaria, CORPOICA. Santa Fe de Bogotá, Colombia, 1994. 29 p.

Identificación, clasificación y análisis de los sistemas de producción agropecuaria de la cuenca hidrográfica del Río Sinú (Parte media y baja). Santa Fe de Bogotá, Colombia, 1998. 104 p.

22 Sandra Lucía Ruiz Rodríguez 